\title{
Digital Eyewear : Synthetic Aperture Focusing in Coded Excitation Scanning Acoustic Microscope
}

\section{Meriläinen, Antti}

IEEE

2019

Meriläinen , A , Hyvönen , J , Salmi , A \& Haeggström , E 2019 , Digital Eyewear : Synthetic Aperture Focusing in Coded Excitation Scanning Acoustic Microscope . in 2019 IEEE International Ultrasonics Symposium (IUS). IEEE International Ultrasonics Symposium, IEEE , pp. 583-585, IEEE International Ultrasonics Symposium , Glasgow , United Kingdom , 06/10/2019 . https://doi.org/10.1109/ULTSYM.2019.8926044

http://hdl.handle.net/10138/326128

https://doi.org/10.1109/ULTSYM.2019.8926044

acceptedVersion

Downloaded from Helda, University of Helsinki institutional repository.

This is an electronic reprint of the original article.

This reprint may differ from the original in pagination and typographic detail.

Please cite the original version. 


\section{Digital Eyewear: Synthetic Aperture Focusing in Coded Excitation Scanning Acoustic Microscope}

\author{
Antti I. Meriläinen
}

Department of Physics

University of Helsinki

Helsinki, Finland

antti.merilainen@helsinki.fi
Jere Hyvönen

Department of Physics

University of Helsinki

Helsinki, Finland
Ari Salmi

Department of Physics

University of Helsinki

Helsinki, Finland
Edward Hæggström

Department of Physics

University of Helsinki

Helsinki, Finland

\begin{abstract}
We developed a synthetic aperture focusing technique (SAFT)-based algorithm to extend the working distance of a traditional scanning acoustic microscopy (SAM) transducer at $400 \mathrm{MHz}$. This algorithm is used to analyze $\mathrm{C}$-scan images of a USAF 1951 resolution sample that is imaged at $50 \mu \mathrm{m}$ defocus and at focus. The comparison of these images shows that this method can enhance the resolution of defocused images. Imaging artefacts caused by this method are discussed.
\end{abstract}

Keywords-Ultrasound imaging, Synthetic aperture, Signal processing, Coded excitation

\section{INTRODUCTION}

Scanning acoustic microscopy (SAM) is a standard method to image sample surfaces at microscopic resolution using a contrast mechanism based on surface mechanics. Parameters affecting the measurements are the acoustic impedance difference of the coupling medium and the surface, sample surface roughness and tilt. At high frequencies the beam is produced with a sapphire lens that are designed for a short working distance, due to the high attenuation of ultrasound. At $400 \mathrm{MHz}$ even $10 \mu \mathrm{m}$ defocus reduces the resolution due to the field shape. This can be solved with multilayer-analysis [1] with the downside of long measurement times. We propose a postprocessing method, based on the synthetic aperture focusing technique (SAFT) [2-4]. This can be used to digitally extend the effective working distance of a traditional focusing ultrasound transducer and to process a single-layer C-scan of uneven samples.

\section{MATERIAL AND METHODS}

\section{A. Scanning acoustic microscope}

Our SAM is based on custom-built hardware, see [5]. Figure 1 shows a schematic picture of our SAM device. Matlab 2017 (Mathworks) was used to control the device via a custom GUIprogram. The SAM operated in pulse-echo-mode, where the transducer first transmitted a TX-signal and then received the echoes from the sample surface. Ion-exchange water was used as immersion medium. The TX-signal is a linear FM-chirp, generated by an arbitrary waveform generator (AWG, M4i.6631-x8, sampling rate $1.25 \mathrm{GS} / \mathrm{s}$, Spectrum Instrumentation). The signal was input to the main amplifier (ZHL-42W, Mini Circuits) via a frequency doubler (ZX90-2-13$\mathrm{S}$, Mini Circuits). RX-signals were pre-amplified by (ZFL-
1000LN+, Mini Circuits) and recorded by a PCIe-oscilloscope (M4i.2233-x8, sampling rate $2.5 \mathrm{GS} / \mathrm{s}$, Spectrum Instrumentation)

A custom-built switch allowed Pulse-echo operation. This switch is based on the HMC435AMS8GE RF-switch circuit. The TX/RX -states are controlled by the AWG.

Since our SAM employed a coded signal, the received signals were cross-correlated with the transmitted signal in Matlab. The cross-correlation increase the SNR, and to compress the linear chirp into a short RF-pulse. The method is described in [5].

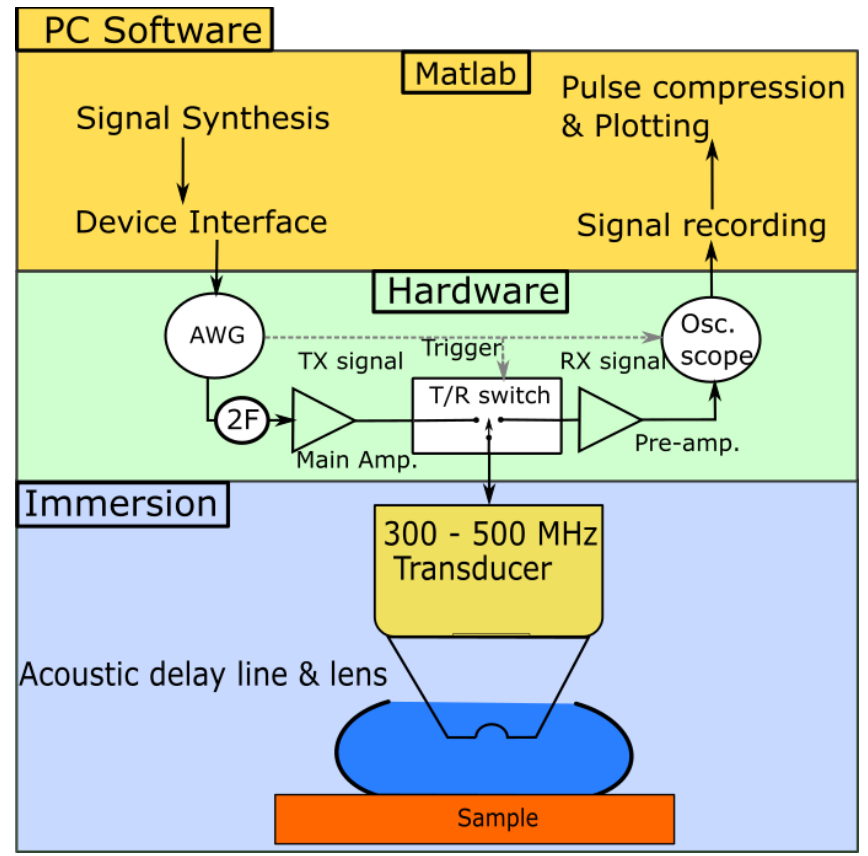

Figure 1, Schematic of the device illustrating the three operational domains: digital, analog, and mechanical.

\section{B. SAFT-algorithm}

The synthetic aperture algorithm is based on a model that describes how the data of a single pulse-echo measurement is delayed. We used a model where the real focus point of transducer's lens, acts as a virtual point source of an ultrasound signal, [4]. This case is illustrated in Fig 2. 
The data pre-processing has the following steps:

1. Cross-correlation of the TX and RX signals

2. Echo time jitter reduction

3. Reduction of the background signal without a reflector

4. FFT of each RX signal (a single pulse-echo measurement)

The 3D complex matrix $\operatorname{Data}(X, Y, f)$ was input to the SAFTrefocusing algorithm:

1. The formation of a delay filter based on the model of the ultrasound beam cone and the average defocus level. The time-of-flight based time delay was transformed into an equivalent frequency dependent phase shift in the Fourier domain. The delay filter is a 3D complex matrix delayFilter(X1,Y1,f) where X1 and Y1 describes the area inside the beam cone.

2. The beam cone area was weighted by a 2D Hann window to reduce side lobes.

3. The delay filter was a kernel for the 2D Convolution: Data(X,Y,f) was 2D-convolved with the delay delayFilter(X1,Y1,f) each frequency component independently.

After the data is ridden from defocus artefacts the C-scan image is formed:

1. SAFT-corrected data is Hilbert transformed resulting in the envelope of the time domain signal.

2. $2^{\text {nd }}$ degree polynomial is fitted to the envelope maximum. Amplitude map is computed from the fitting parameters.

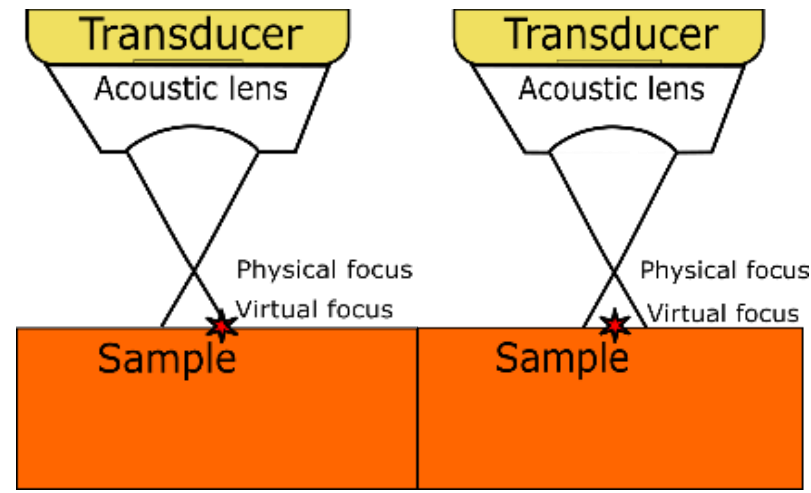

Figure 2, Schematic of the focusing beam formed by a spherical acoustic lens. The star resembles the virtual focus.

\section{Test measurements}

We used a USAF 1951 (R1DS1P, Thorlabs) resolution test sample as a target. A C-Scan was measured from groups 6 and 7. Scans were done first along the X-axis on-the-fly and then the
$\mathrm{Y}$ axis was shifted. Next the primary axis was flipped, and all scans was repeated by taking the $\mathrm{Y}$-axis on-the-fly while the $\mathrm{X}$ axis was shifted. This was done to detect possible image distortion caused by the translation stages. C-scans were performed at $-50 \mu \mathrm{m}$ defocus and in focus.

\section{RESULTS}

\section{A. SAFT Off-Focus Correction}
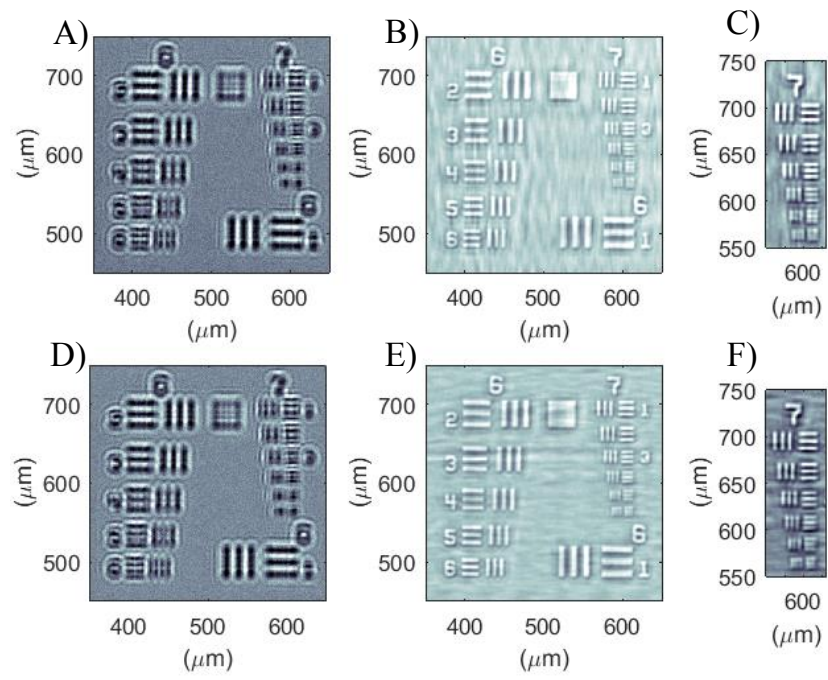

Figure 3, SAFT refocusing, $A$ and D are XY and YX C-scans of the USAF 1951 resolution sample, respectively. Scans were obtained with $-50 \mu m$ defocus. $B$ and $C$ are $S A F T$-corrected images of $A$ and $B$, respectively. $C$ and $F$ are zoom-in of the group 7 demonstrating the resolution after SAFT-correction.

\section{B. In-focus C-Scan}
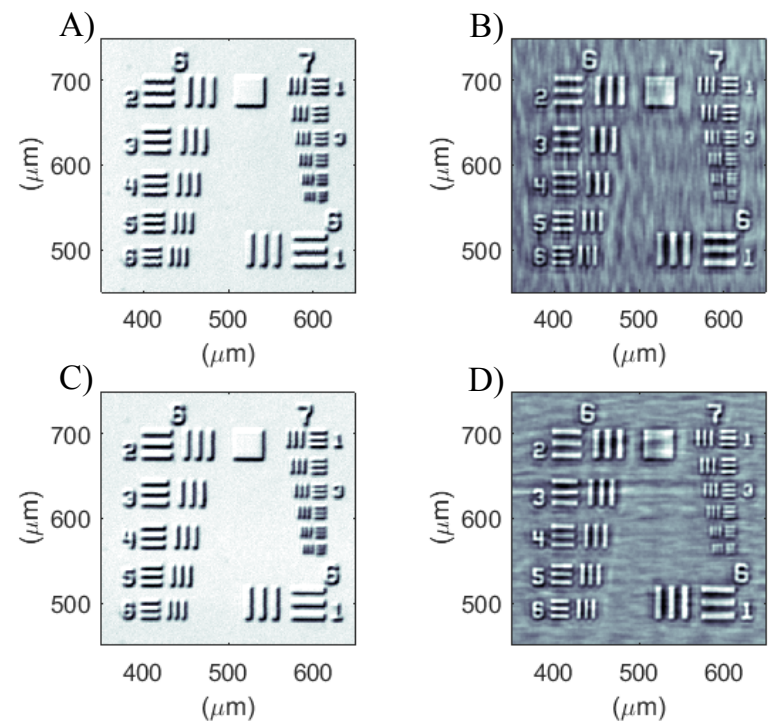

Figure 4, imaging artefacts, $A$ and $C$ are $X Y$ and $Y X$ in-focus $C$-scans of the USAF 1951 resolution sample, respectively. $B$ and D (Scan direction is swapped between the images) are SAFT corrections of same area with 50 m defocus. 


\section{DISCUSSION}

Figure 3: Lateral resolution and contrast can be recovered by SAFT. This shows that resolution is not lost even if the target is $-50 \mu \mathrm{m}$ defocused, i.e. $15 \%$ of the working distance too far away. In normal operation the depth of focus is $\pm 5 \mu \mathrm{m}$, so compared to this the depth of focus has increased by $10 \mathrm{x}$.

Artefacts: In figure 4 one can compare in-focus and SAFT corrected $\mathrm{C}$-scan images. The main difference is visible at the center of the images where the target is clear soda lime glass: In focus the background is smooth white, whereas the SAFTcorrected image shows a random line pattern. The pattern is orientated according to main scanning axis. For the in-focus images the main scanning axis has been swapped, but the swap has no effect to the C-scan images (Fig $2 \mathrm{~A}$ and $\mathrm{C}$ are similar).

This method may find use with samples featuring large surface height variation - instead of performing multilayer analysis [5] one could enhance a single scan image. The amplitude images produced by this method contain some imaging artifacts not detailed here. However, due to artefacts the amplitude values produced by this method are less well defined than amplitude values of the in-focus image. To use this method to calculate absolute unit values from the images [6] one should proceed with care to retain comparability between different measurements and traceability to international standards. This method has so far been shown to work in surface imaging with no internal echoes from the sample.

\section{CONCLUSION}

We showed that with a SAFT algorithm we can digitally extend the working distance of a transducer by reconstructing the image from defocused echoes. The achieved extension of working distance was $15 \%$. The depth of the virtual focus was estimated to be 10x of the non-enhanced one.

\section{REFERENCES}

[1] K. Raum, K. V. Jenderka, A. Klemenz and J. Brandt, "Multilayer analysis: quantitative scanning acoustic microscopy for tissue characterization at a microscopic scale," in IEEE Transactions on Ultrasonics, Ferroelectrics, and Frequency Control, vol. 50, no. 5, pp. 507-516, May 2003.

[2] T. Stepinski, "An implementation of synthetic aperture focusing technique in frequency domain," (in English), Ieee Transactions on Ultrasonics Ferroelectrics and Frequency Control, vol. 54, no. 7, pp. 1399-1408, Jul 2007.

[3] T. Stepinski and F. Lingvall, "Synthetic aperture focusing techniques for ultrasonic imaging of solid objects," in 8th European Conference on Synthetic Aperture Radar, 2010, pp. 1-4: VDE.

[4] T. Olofsson, M. H. Skjelvareid, and A. Barkefors, "Ultrasonic imaging of immersed objects using migration techniques," in 8th European Conference on Synthetic Aperture Radar, 2010, pp. 1-4: VDE.

[5] A. Barkefors, "3D Synthetic Aperture Technique for Ultrasonic Imaging," ed, 2010

[6] J. Hyvönen, A. Meriläinen, A. Salmi, and E. Hæggström, "Three Megapixel Ultrasonic Microscope Imaging ", IEEE IUS 2019, Glasgow 